\title{
WIP: Supporting Faculty Developers' Engagement with Disciplinary Perspectives
}

\section{Mr. Richard J. Aleong, Purdue University-Main Campus, West Lafayette (College of Engineering)}

Richard J. Aleong is a Ph.D. candidate in the School of Engineering Education at Purdue University. He received his M.A.Sc. and B.Sc.E in Mechanical and Materials Engineering from Queen's University, Kingston, Canada. His research interests are focused on integrative thinking and design, interdisciplinary collaboration, and educational development to support students' personal and professional learning and growth. 


\section{WIP: Supporting Faculty Developers' Engagement with Disciplinary Perspectives}

\section{Introduction}

This work in progress presents three ways of approaching the analysis of an empirical research study that explores how faculty developers engage with disciplinary perspectives. As a core element of interdisciplinary work, disciplinary perspectives represent the ways individuals may see and approach a problem based on their unique disciplinary background and training. This paper aims to evoke reflection on faculty development as an interdisciplinary practice with implications for the professional development and identity of faculty developers.

\section{Faculty Development as Interdisciplinary Work}

In the work of faculty development, faculty developers bring their own disciplinary backgrounds to their roles, collaborate across disciplines, and operate at disciplinary borders within institution-wide and discipline-specific academic units [1]. In this project, faculty development is framed as interdisciplinary work where faculty developers work to integrate multiple perspectives towards creating educational solutions and supporting faculty and graduate students in the development of their teaching and learning practice. Within these interdisciplinary interactions, challenges and conflict may arise because academic disciplines have different ways of seeing problems and different methods for problem solving. Therefore, it is important to understand how disciplines shape the perspectives that faculty developers bring to their collaborative interactions [2]. Furthermore, it is important to understand how faculty developers may be supported in developing their interdisciplinary ways of working and shared identities that leverage disciplinary perspectives.

\section{Disciplinary Perspectives}

In this research, the phenomenon of interest is disciplinary perspectives as an object that faculty developers hold and engage with through their interdisciplinary interactions. Repko and Szostak [3] defined disciplinary perspectives as a "discipline's view of reality in a general sense that embraces and in turn reflects the ensemble of its defining elements that include phenomena, epistemology, assumptions, concepts, theories, and methods" (p. 32). Individuals engage with disciplinary perspectives in different ways depending on the nature of the interdisciplinary task and to the extent of integration required for a desirable outcome [4], [5].

\section{Methods}

To explore faculty developer's engagement with disciplinary perspectives, semistructured interviews were conducted with faculty developers across the United States and Canada. The focus of the interview was for participants to describe their experiences as a faculty developer and ways they may have engaged in interdisciplinary collaboration. Initial analysis of the findings is currently underway, and this paper aims to foster critical conversation and community feedback about opportunities, further questions, and implications for consideration as 
the research progresses. In the following section, I offer three frames for analysis that provide three ways for exploring the data that focuses on interdisciplinary practices of faculty developers.

\section{Interdisciplinary Practices}

This research aims to contribute to an understanding of interdisciplinary practices for collaboration in the context of faculty development. Therefore, I present three frames for analysis that provide a way of seeing how disciplinary perspectives may appear in the work of faculty development. The act of framing presents opportunities for expanding our understanding of a problem by enabling different ways of seeing and thinking about the problem see [6]. Here, I use framing and reframing as an analytical tool to develop a shared understanding of my phenomenon of interest and as different approaches to make meaning of my data. In this way, I propose three initial frames to sensitize myself to the data. These frames emphasize ways of looking at the data with a focus on aspects within individual participants, relationships with others, and how disciplinary perspectives may fit in a broader social system. In the following section, I outline these three frames and provide an illustration from participant data.

\section{Frame 1: Critical self-awareness of one's disciplinary perspective}

This framing approach considers how faculty developer's may reflectively engage with their own disciplinary perspective and how it might inform their practice. This frame captures ways of looking at elements within the individual participant. The following participant quote, demonstrates how a participant's critical awareness of their assumptions influences their approach when they are faced in a new situation:

"So, depending on the discipline, I think I know how to approach it. But then I am always surprised, right? Because I'm making some assumptions and I've got to keep checking my assumptions, too.” (Brooke)

\section{Frame 2: Eliciting and utilizing the disciplinary perspectives of others}

This frame emphasizes how faculty developers may draw on the disciplinary perspectives of others to inform their work. This frame highlights the possibility for seeing relationships between the faculty developer, disciplinary perspectives as a phenomenon, and working with others. The following participant quote illustrates this faculty developer's orientation towards elicitation and utilization of faculty perspectives.

"My role [as an educational developer] is really to promote [faculty's] dialogue amongst themselves or to promote that individual faculty member's development of their teaching and learning to advance their practice, but that needs to come from a place of excellence that's defined by them more than it is by me" (Allie)

\section{Frame 3: Seeing disciplinary perspectives within social, cultural, and political contexts}

This frame aims to capture how disciplinary perspectives exists within broader social, cultural, and political contexts of higher education and faculty development work. This participant 
identified "local culture" as an important aspect that influences their interactions with faculty, along with an understanding of different ways of thinking and doing:

"In different ways, those three domains, kind of ways of thinking, ways of doing, and then the local culture, all come into play with how [faculty] are going to interact with you as an educational developer and the kind of programming, or delivery, or conversation that you want to have with them" (Wendy)

Taken together, these three frames may highlight points of interest and opportunities for further meaning making of the data. These frames offer an initial lens to explore relationships within and between actors, the phenomenon of interest, and how these two elements are situated within the context of faculty development work. An understanding of faculty developers' experiences with disciplinary perspectives aims to contribute to the different ways faculty developers may reflect on, recognize, and elicit disciplinary perspectives in collaborative practice.

\section{Discussion: Critical Questions for Growing Faculty Development}

This paper aims to foster larger conversations and critical questions facing the faculty development community: What does it mean for faculty developers to be and become interdisciplinary? How can faculty developers operate in ways that support interdisciplinary collaboration? With a view of faculty development as a disciplinary and interdisciplinary activity, faculty developers may be positioned to explore and articulate faculty development's own disciplinary perspectives towards the growth as a professional field.

In engineering education, literature has pointed to critical conversations of the development of engineering education research as a discipline, community, or field, pointing to challenges and opportunities for growth see [7]. For faculty development, the structure of disciplines in higher education may evoke boundaries and exclusivity, but may also offer benefits of building legitimacy and recognition of the field. This tension will need to be continually navigated and negotiated among faculty developers as we aim to inclusively grow the field while establishing expertise in our professional work. With an understanding of interdisciplinary practice and how faculty developers engage in interdisciplinary work, an interdisciplinary professional frame may serve to integrate these competing values and provide shared professional identities and ways of working. I offer the following questions for critical reflection:

- What professional expertise or ways of working hold us together?

- What would it mean to grow the field of faculty development as interdisciplinary?

- How do faculty developers disciplinary and interdisciplinary identities inform their faculty development practice?

Responses to these questions are intimately interconnected and mutually supportive. As faculty developers grow in their identity, this identity and professional discipline brings a stronger sense of who we are, what we know, and how we can contribute. In this paper, I offer an interdisciplinary perspective toward this effort, grounded in an understanding of individual disciplinary perspectives. 


\section{Conclusion}

In this paper, I have offered three preliminary frames for thinking about engagement with disciplinary perspectives and how these frames may guide further research analysis into the various ways faculty developer's experience engagement with disciplinary perspectives. I have also highlighted areas of opportunity for faculty development as a professional field and the role that disciplinary perspectives may play in understanding and advancing these areas. Understanding faculty developers' interactions and relationships with disciplinary perspectives offers one way to build shared professional identities and highlight our shared disciplinary expertise in the work of faculty development.

This paper aims to evoke reflection on faculty development as an interdisciplinary practice with implications for the professional development and identity formation of faculty developers. The preferred presentation method is for a lightning talk where the author will be able to engage community feedback and discussion on the usefulness of the research and the affordances and challenges of adopting interdisciplinary approaches in faculty development.

\section{References}

[1] L. McAlpine and R. Harris, "Lessons learned: Faculty developer and engineer working as faculty development colleagues," Int. J. Acad. Dev., vol. 4, no. 1, pp. 11-17, May 1999, doi: 10.1080/1360144990040103.

[2] D. Little, D. A. Green, and C. Hoption, "A lasting impression: the influence of prior disciplines on educational developers' research," Int. J. Acad. Dev., pp. 1-15, Apr. 2018, doi: 10.1080/1360144X.2018.1458617.

[3] A. Repko and R. Szostak, Interdisciplinary research: Process and theory. Thousands Oaks, CA: Sage, 2017.

[4] J. B. Holbrook, "What is interdisciplinary communication? Reflections on the very idea of disciplinary integration," Synthese, vol. 190, no. 11, pp. 1865-1879, Jul. 2013, doi: 10.1007/s11229-012-0179-7.

[5] M. O’Rourke, S. Crowley, and C. Gonnerman, "On the nature of cross-disciplinary integration: A philosophical framework," Stud. Hist. Philos. Sci. Part C Stud. Hist. Philos. Biol. Biomed. Sci., vol. 56, pp. 62-70, Apr. 2016, doi: 10.1016/j.shpsc.2015.10.003.

[6] D. Ellis, "Changing the lens: The role of reframing in educational development," Improve Acad., vol. 37, no. 1, pp. 142-150, Feb. 2018, doi: 10.1002/tia2.20067.

[7] B. K. Jesiek, L. K. Newswander, and M. Borrego, "Engineering education research: Discipline, community, or field?," J. Eng. Educ., vol. 98, no. 1, pp. 39-52, 2009, doi: 10.1002/j.2168-9830.2009.tb01004.x. 